127 Maria João Costa Pacheco

Márcia Patrícia Costa Pacheco

Mónica Oliveira Medeiros Botelho

Pedro Rodrigues Amaral

Ricardo Jorge Correia Pacheco

\author{
Correspondencia: \\ Ricardo Pacheco \\ Avenida D. João III, Bloco 1-E, 3o Direito \\ 9500-310, São Pedro \\ Ponta Delgada, São Miguel (Açores) \\ Tfno.: +351962610415 \\ E-mail: ricardocalheta@gmail.com
}

\section{Anciano que cuida a anciano}

25

\section{RESUMEN}

El envejecimiento es un proceso complejo, continuo, irreversible y dinámico al que la población portuguesa no es ajena. Con la longevidad, los ancianos son cada vez menos autónomos, más dependientes de cuidados de terceros, y estos cuidados aparecen, cada vez más, proporcionados por otro anciano.

Actualmente los enfermeros deben dar una atención especial a este fenómeno para suministrar un apoyo mejor a los cuidadores ancianos que cuidan a otros ancianos, también ellos personas con problemas geriátricos. Deberán proporcionar una mayor dedicación a la adquisición y mantenimiento de este papel para promover la autonomía y minimizar la dependencia del anciano cuidado.

\section{PALABRAS CLAVE}

Envejecimiento, apoyo psicológico, educación/información, cuidador informal anciano.

\author{
Elderly caring for elderly
}

Este trabajo, titulado "Anciano que cuida a anciano", surge en un contexto académico realizado por estudiantes de la $13^{a}$ Maestría (grupo B) en "Ciencias de la Enfermería" del Instituto de Ciencias Biomédicas Abel Salazar de la Universidad de Oporto (Oporto-Portugal), que tiene lugar en la Escuela Superior de Enfermería de Ponta Delgada (Azores). Tiene como objetivo reflexionar sobre los sentimientos, dificultades de los cuidadores de ancianos (edad superior o igual a los 65 años) que cuidan a otros ancianos (edad superior a igual a 65 años). Este artículo se presentó en el primer semestre de la respectiva maestría, actividad que está dentro del plan de actividades pedagógicas.

\section{INTRODUCCIÓN}

En la actualidad, el envejecimiento ha ganado una importancia especial en nuestro cotidiano social que no se puede olvidar, estando presente en las preocupaciones sociopolíticas del mundo occidental. Según Costa (1), los epidemiólogos definen un hecho actual que es el de la transición demográfica relacionada con dos factores: la progresiva disminución de las tasas de natalidad y de las tasas de mortalidad (y el consecuente aumento de la esperanza de vida) con repercusiones económicas y sociales. De acuerdo con el Instituto Nacional de Estadística portugués, citado por Moniz (2), "[...] hacia 2020 las personas con más de 65 años representarán el $18,1 \%$ de la población portuguesa”.

Con este escenario demográfico como telón de fondo, las profesiones relacionadas con la salud, en particular la enfermería, empiezan a reflexionar sobre los temas subya-

\section{SUMMARY}

Aging is a complex process, continuous and irreversible, a phenomenon to which Portuguese population is not stranger. With longevity the elderly becomes less autonomous, depending on the case of others, starting to emerge a growing number of elderly much more elderly, many of them taking care of another elder.

In the present nurses should give special attention to this phenomenon in order to provide better support to the elderly caregivers of the elderly, who also have geriatrics problems, proving a greater satisfaction in the acquisition and maintenance of this role and in this way promoting self-governing and minimizing the dependency of the cared elder.

\section{KEY WORDS}

Aging, psychological support, education/information, social system of assistance, profile of the informal elder caregiver. 
Tabla 1. Número de la población anciana por grupos de edad, por isla, de la RAA, y porcentaje teniendo en cuenta la población de la isla

\begin{tabular}{|c|c|c|c|c|c|c|c|}
\hline Isla & $\geq 65$ años & $\geq 70$ años & $\geq 75$ años & $\geq 80$ años & $\geq 85$ años & Total & Porcentaje \\
\hline $\mathrm{S}^{\text {ta }}$ Maria & 253 & 178 & 141 & 79 & 50 & 701 & 12,57 \\
\hline S. Miguel & 4.662 & 3.743 & 3.151 & 1.646 & 1.117 & 14.319 & 10,88 \\
\hline Terceira & 2.356 & 2.109 & 1.546 & 1.007 & 677 & 7.695 & 13,78 \\
\hline Faial & 609 & 592 & 484 & 334 & 236 & 2.255 & 14,97 \\
\hline Pico & 871 & 728 & 572 & 388 & 280 & 2.839 & 19,17 \\
\hline S. Jorge & 554 & 485 & 304 & 199 & 158 & 1.700 & 17,57 \\
\hline Graciosa & 320 & 277 & 211 & 124 & 82 & 1.014 & 21,21 \\
\hline Flores & 189 & 191 & 165 & 104 & 66 & 715 & 17,90 \\
\hline Corvo & 17 & 21 & 19 & 18 & 6 & 81 & 19,06 \\
\hline Total & 9.831 & 8.324 & 6.593 & 3.899 & 2.672 & 31.319 & 12,95 \\
\hline
\end{tabular}

Tabla 2. Número total de ancianos que reciben cuidados y número de cuidadores ancianos por unidad de salud del centro de salud de Ponta Delgada

\begin{tabular}{|l|l|l|}
\hline & $\begin{array}{l}\text { Número } \\
\text { de ancianos que } \\
\text { reciben cuidados } \\
\text { por el equipo de } \\
\text { enfermería } \\
\text { a domicilio }\end{array}$ & $\begin{array}{l}\text { Número } \\
\text { de ancianos con } \\
\text { cuidador anciano }\end{array}$ \\
de salud & 93 & 16 \\
\hline Arrifes & 74 & 7 \\
Capelas & 107 & 22 \\
Feteiras & 36 & 10 \\
Matriz & 40 & 8 \\
São José & 49 & 3 \\
Santo António & 399 & $66(16,5 \%)$ \\
\hline Total & \multicolumn{2}{|l}{}
\end{tabular}

Heroísmo y Horta) fue del 36,61\%, siendo el porcentaje del hospital de Ponta Delgada del 35,22\%. Este trabajo tiene como objetivo reflexionar sobre un fenómeno que se presenta, en un futuro próximo, como un verdadero desafío para la enfermería como profesión y disciplina: un anciano que cuida a otro anciano. Este ejercicio se ha basado en la revisión sistemática de la bibliografía, e inten- ta descubrir el perfil del anciano cuidador en el municipio de Ponta Delgada de la RAA. De esta manera nos acercamos a la preocupación de Paúl (5) cuando afirma que no sólo tenemos que conocer al cuidador como tenemos que intervenir con él, anticipándonos a sus necesidades y apoyando su esfuerzo para evitar desequilibrios perjudiciales para él y para el anciano que recibe los cuidados.

\section{Cuidador informal}

Cuidar a alguien ha sido siempre, desde los comienzos de la humanidad, una constante preocupación del hombre. Collière (6) lo define como " $[\ldots]$ un acto individual que nos prestamos a nosotros mismos desde que somos autónomos, pero es también un acto de reciprocidad que prestamos a toda persona que, temporal o definitivamente, tiene la necesidad de ayuda para realizar sus necesidades vitales".

La calidad de vida de las personas ancianas dependientes depende muchas veces y casi exclusivamente de la calidad y formación de aquellos que les cuidan, ya sean cuidadores informales o formales, siendo los cuidadores informales aquellos que prestan cuidados sin formación específica. Haciendo referencia a Vieira, citado por Zimerman (7), son cuidadores "[...] las personas que se dedican a la tarea de cuidar a un anciano [...]". Una vez que en la bibliografía consultada no ha sido posible encontrar una definición de cuidador informal anciano, de otro anciano, creemos que se podrá definir como aquel que, con una edad igual o superior a

Tabla 3. Edad de los cuidadores informales ancianos, distribuidos por unidades de salud

\begin{tabular}{|l|l|l|l|l|l|l|l|}
\hline Unidad de salud & Feteiras & Santo António & Capelas & Arrifes & Matriz & São José \\
\hline $\begin{array}{l}\text { Edad } \\
\text { (años) }\end{array}$ & $\begin{array}{l}\text { Número } \\
\text { de casos }\end{array}$ & $\begin{array}{l}\text { Número } \\
\text { de casos }\end{array}$ & $\begin{array}{l}\text { Número } \\
\text { de casos }\end{array}$ & $\begin{array}{l}\text { Número } \\
\text { de casos }\end{array}$ & $\begin{array}{l}\text { Número } \\
\text { de casos }\end{array}$ & $\begin{array}{l}\text { Número } \\
\text { de casos }\end{array}$ & Total \\
\hline $65-70$ & 6 & 1 & 3 & 3 & 4 & 6 & 23 \\
$71-75$ & 8 & 2 & 2 & 2 & 2 & 1 & 17 \\
$76-80$ & 4 & 0 & 2 & 7 & 1 & 0 & 14 \\
$81-85$ & 4 & 0 & 0 & 4 & 3 & 8 & 66 \\
Total & 22 & 3 & 7 & 16 & 10 & 8 \\
\hline
\end{tabular}


Tabla 4. Sexo de los cuidadores informales ancianos, distribuidos por unidades de salud

\begin{tabular}{|l|l|l|l|l|l|l|l|}
\hline Unidad de salud & Feteiras & Santo António & Capelas & Arrifes & Matriz & São José \\
\hline Sexo & $\begin{array}{l}\text { Número } \\
\text { de casos }\end{array}$ & $\begin{array}{l}\text { Número } \\
\text { de casos }\end{array}$ & $\begin{array}{l}\text { Número } \\
\text { de casos }\end{array}$ & $\begin{array}{l}\text { Número } \\
\text { de casos }\end{array}$ & $\begin{array}{l}\text { Número } \\
\text { de casos }\end{array}$ & $\begin{array}{l}\text { Número } \\
\text { de casos }\end{array}$ & Total \\
\hline Femenino & 19 & 2 & 5 & 11 & 6 & 8 & 51 \\
Masculino & 3 & 1 & 2 & 5 & 4 & 0 & 15 \\
\hline Total & 22 & 3 & 7 & 16 & 10 & 8 & 66 \\
\hline
\end{tabular}

Tabla 5. Grado de parentesco de los cuidadores informales ancianos con los ancianos que atendían, distribuidos por unidades de salud

\begin{tabular}{|l|l|l|l|l|l|l|l|}
\hline Unidad de salud & Feteiras & Santo António & Capelas & Arrifes & Matriz & São José & \\
\cline { 1 - 5 } $\begin{array}{l}\text { Grado } \\
\text { de parentesco }\end{array}$ & $\begin{array}{l}\text { Número } \\
\text { de casos }\end{array}$ & $\begin{array}{l}\text { Número } \\
\text { de casos }\end{array}$ & $\begin{array}{l}\text { Número } \\
\text { de casos }\end{array}$ & $\begin{array}{l}\text { Número } \\
\text { de casos }\end{array}$ & $\begin{array}{l}\text { Número } \\
\text { de casos }\end{array}$ & $\begin{array}{l}\text { Número } \\
\text { de casos }\end{array}$ & Total \\
\hline Marido & 3 & 1 & 2 & 5 & 4 & 0 & 15 \\
Esposa & 18 & 1 & 4 & 8 & 4 & 7 & 42 \\
Amiga & 1 & - & 1 & - & 0 & - & 2 \\
Hermana & - & 1 & - & 2 & 2 & 1 & 6 \\
Hija & - & - & - & 1 & 0 & - & 1 \\
\cline { 1 - 5 } Total & 22 & 3 & 7 & 16 & 10 & 8 & 66 \\
\hline
\end{tabular}

Tabla 6. Profesión de los cuidadores informales ancianos, distribuidos por unidades de salud

\begin{tabular}{|c|c|c|c|c|c|c|c|}
\hline Unidad de salud & Feteiras & Santo António & Capelas & Arrifes & Matriz & São José & \\
\hline $\begin{array}{l}\text { Situación } \\
\text { profesional }\end{array}$ & $\begin{array}{l}\text { Número } \\
\text { de casos }\end{array}$ & $\begin{array}{l}\text { Número } \\
\text { de casos }\end{array}$ & $\begin{array}{l}\text { Número } \\
\text { de casos }\end{array}$ & $\begin{array}{l}\text { Número } \\
\text { de casos }\end{array}$ & $\begin{array}{l}\text { Número } \\
\text { de casos }\end{array}$ & $\begin{array}{l}\text { Número } \\
\text { de casos }\end{array}$ & Total \\
\hline Jubilado & 8 & 1 & 3 & 5 & 6 & - & 23 \\
\hline Ama de casa & 13 & 1 & 4 & 11 & 4 & 8 & 41 \\
\hline Pensionista & 1 & - & - & - & - & - & 1 \\
\hline Agricultor & - & 1 & - & - & - & - & 1 \\
\hline Total & 22 & 3 & 7 & 16 & 10 & 8 & 66 \\
\hline
\end{tabular}

Tabla 7. Escolaridad de los cuidadores informales ancianos, distribuidos por unidades de salud

\begin{tabular}{|l|l|l|l|l|l|l|l|}
\hline Unidad de salud & Feteiras & Santo António & Capelas & Arrifes & Matriz & São José & \\
\hline Escolaridad & $\begin{array}{l}\text { Número } \\
\text { de casos }\end{array}$ & $\begin{array}{l}\text { Número } \\
\text { de casos }\end{array}$ & $\begin{array}{l}\text { Número } \\
\text { de casos }\end{array}$ & $\begin{array}{l}\text { Número } \\
\text { de casos }\end{array}$ & $\begin{array}{l}\text { Número } \\
\text { de casos }\end{array}$ & $\begin{array}{l}\text { Número } \\
\text { de casos }\end{array}$ & \multirow{2}{*}{ Total } \\
\hline No posee & 9 & 2 & 4 & 10 & 0 & 2 & 27 \\
$3^{\text {a clase }}$ & 3 & 0 & 2 & 1 & 3 & 2 & 11 \\
$1^{\text {er ciclo completo }}$ & 10 & 1 & 1 & 5 & 7 & 3 & 27 \\
Secundaria & 0 & 0 & 0 & 0 & 0 & 1 & 1 \\
\hline Total & 22 & 3 & 7 & 16 & 10 & 8 & 66 \\
\hline
\end{tabular}

65 años, presta cuidados, sin formación específica, a otra persona con edad igual o superior a los 65 años.

En el dictamen de Cohen y Eisdorfer citado por Lurdes y Storani, en Netto (8), la motivación para cuidar a alguien se basa en cuatro razones principales: amor, gratitud, moralidad y voluntad propia de cuidar. Para el cuidador informal anciano, cuidar supone consecuencias para la salud del propio cuidador, consecuencias que van desde el aspecto físico hasta el social, pasando por el emocional, psicológico e incluso existencial.
Muchas veces el cuidador de anciano dependiente es el cónyuge, hecho que supone problemas de diferente orden ya que los dos (anciano y cuidador) pertenecen al mismo grupo de edad. El Consejo Económico y Social (9) dice que "[...] la situación de las parejas ancianas se caracteriza, en repetidas ocasiones, por el apoyo recíproco que se da dentro de un frágil equilibrio y de un lento deterioro de los dos, cada uno apoyándose en el otro en función de lo que cada uno puede soportar todavía [...]". Para éste, en los cuidadores en Portugal es frecuente el "Exceso de cansancio, fatiga general, dolo- 
Costa Pacheco, M.J.; Costa Pacheco, M.P.; Medeiros Botelho, M.O.; Rodrigues Amaral, P.; Correia Pacheco, R.J.: ANCIANO QUE CUIDA A ANCIANO

Tabla 8. Apoyos sociales que de los cuidadores informales ancianos, distribuidos

por unidades de salud

\begin{tabular}{|c|c|c|c|c|c|c|c|}
\hline Unidad de salud & Feteiras & Santo António & Capelas & Arrifes & Matriz & São José & \\
\hline $\begin{array}{l}\text { Apoyo } \\
\text { social }\end{array}$ & $\begin{array}{l}\text { Número } \\
\text { de casos }\end{array}$ & $\begin{array}{l}\text { Número } \\
\text { de casos }\end{array}$ & $\begin{array}{l}\text { Número } \\
\text { de casos }\end{array}$ & $\begin{array}{l}\text { Número } \\
\text { de casos }\end{array}$ & $\begin{array}{l}\text { Número } \\
\text { de casos }\end{array}$ & $\begin{array}{l}\text { Número } \\
\text { de casos }\end{array}$ & Total \\
\hline No disfruta & 18 & 2 & 5 & 13 & 8 & 6 & 52 \\
\hline $\begin{array}{l}\text { Disfruta: cuidados d } \\
\text { higiene, limpieza } \\
\text { de la casa }\end{array}$ & 4 & - & - & - & 2 & 1 & 7 \\
\hline $\begin{array}{l}\text { Disfruta: apoyo } \\
\text { monetario para } \\
\text { comprar } \\
\text { medicamentos/ } \\
\text { infraestruturas }\end{array}$ & - & 1 & - & - & - & 1 & 2 \\
\hline $\begin{array}{l}\text { Disfruta: cuidados } \\
\text { de higiene }\end{array}$ & - & - & 2 & - & 2 & 1 & 5 \\
\hline $\begin{array}{l}\text { Disfruta: apoyo } \\
\text { a } 3^{\mathrm{a}} \text { persona }\end{array}$ & - & - & - & 3 & - & - & 3 \\
\hline Total & 22 & 3 & 7 & 16 & 12 & 9 & 69 \\
\hline
\end{tabular}

Tabla 9. Historial clínico de los cuidadores informales ancianos, distribuidos por unidades de salud

\begin{tabular}{|c|c|c|c|c|c|c|c|}
\hline Unidad de salud & Feteiras & Santo António & Capelas & Arrifes & Matriz & São José & \\
\hline $\begin{array}{l}\text { Historial } \\
\text { clínico }\end{array}$ & $\begin{array}{l}\text { Número } \\
\text { de casos }\end{array}$ & $\begin{array}{l}\text { Número } \\
\text { de casos }\end{array}$ & $\begin{array}{l}\text { Número } \\
\text { de casos }\end{array}$ & $\begin{array}{l}\text { Número } \\
\text { de casos }\end{array}$ & $\begin{array}{l}\text { Número } \\
\text { de casos }\end{array}$ & $\begin{array}{l}\text { Número } \\
\text { de casos }\end{array}$ & Total \\
\hline Ansiedad & 1 & - & - & - & - & 3 & 4 \\
\hline Bocio & 1 & - & - & - & - & - & 1 \\
\hline Cardíaco & 5 & 1 & - & 6 & 1 & 1 & 14 \\
\hline Cataratas/ptosis & & & & & & & \\
\hline palpebral & - & - & - & 1 & - & 1 & 2 \\
\hline Circulatorio & 3 & 0 & - & 2 & - & - & 5 \\
\hline Depresión & 4 & - & 1 & 1 & - & - & 6 \\
\hline Diabetes & 4 & 2 & 2 & 5 & 1 & 2 & 16 \\
\hline Digestivo & 4 & 1 & 2 & 5 & - & - & 12 \\
\hline Ginecológico & 2 & 0 & 2 & 5 & - & - & 9 \\
\hline Hematológico & 1 & 0 & - & - & - & - & 1 \\
\hline Hemorroides & - & - & 1 & - & - & - & 1 \\
\hline Hernia inguinal & - & 1 & - & - & - & - & 1 \\
\hline Hipercolesterolemia & 1 & - & 1 & - & - & - & 2 \\
\hline Hipertensión arterial & 18 & 2 & 3 & 5 & 1 & 2 & 31 \\
\hline Hipertiroidismo & - & - & - & 1 & - & - & 1 \\
\hline Insomnio & 2 & - & - & 1 & - & - & 3 \\
\hline Mastectomizado & - & 1 & - & - & - & - & 1 \\
\hline Neurológico & 1 & 0 & 1 & - & - & - & 2 \\
\hline Osteomuscular & 9 & 2 & 3 & 9 & 3 & 2 & 28 \\
\hline Renal & 0 & 0 & 2 & 1 & - & - & 3 \\
\hline Respiratorio & 3 & 0 & - & 2 & 1 & - & 6 \\
\hline Sinusitis & - & 1 & - & - & - & - & 1 \\
\hline Urológico & 2 & 1 & - & 3 & - & - & 6 \\
\hline Total & 61 & 12 & 18 & 47 & 7 & 11 & 156 \\
\hline
\end{tabular}

res de espalda, agotamiento físico y mental, disminución de las fuerzas y de la resistencia, nerviosismo, irritabilidad, ansiedad, insomnio, estado depresivo [...]". Hace referencia también a que son varios los sentimientos que podrá originar el hecho de cuidar a ancianos: cul- pa; ternura, compasión; tristeza o impotencia; angustia por la muerte, ante la suya y la del otro y el efecto espe$j o$. También el hecho de que el cuidador sea anciano provoca que se pregunte acerca de quién continuará cuidando al otro anciano en el caso de que muera primero 


\begin{tabular}{|c|c|c|c|c|c|c|c|}
\hline Unidad de salud & Feteiras & Santo António & Capelas & Arrifes & Matriz & São José & \\
\hline $\begin{array}{l}\text { Actividades de vida } \\
\text { cotidiana }\end{array}$ & $\begin{array}{l}\text { Número } \\
\text { de casos }\end{array}$ & $\begin{array}{l}\text { Número } \\
\text { de casos }\end{array}$ & $\begin{array}{l}\text { Número } \\
\text { de casos }\end{array}$ & $\begin{array}{l}\text { Número } \\
\text { de casos }\end{array}$ & $\begin{array}{l}\text { Número } \\
\text { de casos }\end{array}$ & $\begin{array}{l}\text { Número } \\
\text { de casos }\end{array}$ & Total \\
\hline $\begin{array}{l}\text { Cuidados de higiene } \\
\text { y conforto totales }\end{array}$ & 16 & 2 & 2 & 9 & 4 & 6 & 39 \\
\hline $\begin{array}{l}\text { Cuidados de higiene } \\
\text { y conforto parciales }\end{array}$ & 3 & 0 & 3 & 5 & 2 & 1 & 14 \\
\hline Alimentación & 15 & 1 & 7 & 11 & 5 & 8 & 47 \\
\hline Eliminación & 7 & 2 & 4 & 9 & 5 & 8 & 35 \\
\hline Movilidad total & 7 & 2 & 5 & 9 & 4 & 7 & 34 \\
\hline Movilidad parcial & 0 & 0 & 0 & 0 & 1 & 1 & 2 \\
\hline Movilidad supervisión & 4 & 0 & 0 & 2 & 2 & - & 8 \\
\hline Total & 52 & 7 & 21 & 45 & 23 & 31 & 179 \\
\hline
\end{tabular}

Tabla 11. Actividades de la vida social cotidiana de los cuidadores informales ancianos, distribuidos por unidades de salud

\begin{tabular}{|c|c|c|c|c|c|c|c|}
\hline Unidad de salud & Feteiras & Santo António & Capelas & Arrifes & Matriz & São José & \\
\hline $\begin{array}{l}\text { Actividades de la } \\
\text { vida social cotidiana }\end{array}$ & $\begin{array}{l}\text { Número } \\
\text { de casos }\end{array}$ & $\begin{array}{l}\text { Número } \\
\text { de casos }\end{array}$ & $\begin{array}{l}\text { Número } \\
\text { de casos }\end{array}$ & $\begin{array}{l}\text { Número } \\
\text { de casos }\end{array}$ & $\begin{array}{l}\text { Número } \\
\text { de casos }\end{array}$ & $\begin{array}{l}\text { Número } \\
\text { de casos }\end{array}$ & Total \\
\hline Cocinar & 20 & 2 & 6 & 11 & 6 & 7 & 52 \\
\hline Limpieza de la casa & 18 & 3 & 6 & 9 & 4 & 6 & 46 \\
\hline Lavandería & 17 & 3 & 6 & 12 & 5 & 7 & 50 \\
\hline $\begin{array}{l}\text { Gestión doméstica: } \\
\text { pagos de cuentas } \\
\text { de la casa }\end{array}$ & 19 & 3 & 7 & 16 & 9 & 7 & 61 \\
\hline $\begin{array}{l}\text { Gestión doméstica: } \\
\text { compras de } \\
\text { alimentación }\end{array}$ & 19 & 3 & 7 & 16 & 9 & 7 & 61 \\
\hline $\begin{array}{l}\text { Gestión doméstica: } \\
\text { compras de } \\
\text { medicación }\end{array}$ & 19 & 3 & 7 & 16 & 9 & 7 & 61 \\
\hline $\begin{array}{l}\text { Preparación } \\
\text { de medicación }\end{array}$ & 16 & 2 & 4 & 11 & 3 & 8 & 44 \\
\hline $\begin{array}{l}\text { Administración } \\
\text { de medicación }\end{array}$ & 17 & 2 & 4 & 11 & 4 & 8 & 46 \\
\hline $\begin{array}{l}\text { Otros cuidados } \\
\text { (evaluación de la } \\
\text { tensión arterial, } \\
\text { glicemia capilar, } \\
\text { realización de curas) }\end{array}$ & 1 & 1 & - & 一 & - & - & 2 \\
\hline Total & 146 & 22 & 47 & 102 & 49 & 57 & 423 \\
\hline
\end{tabular}

o si enferma o pierde autonomía y es incapaz de continuar cuidando.

\section{Contribuciones del enfermero a la relación} con el cuidador informal anciano

Para que podamos cuidar verdaderamente tenemos que reconocer en el anciano la fragilidad de donde surge la necesidad de cuidar y la capacidad de este anciano para participar en los cuidados. La clasificación de Soldevilla Agreda (3) caracteriza a los destinatarios de la enfermería geronto- lógica clasificándolos en personas ancianas sanas: individuos independientes y capaces de satisfacer sus necesidades dentro de sus limitaciones, impuestas por el factor de la edad; y las personas mayores frágiles o de riesgo: ancianos con alto riesgo de perder su autonomía, sufrir complicaciones, ingresos en instituciones o morir, y los enfermos geriátricos.

Para conseguir una evaluación cabal del cuidador anciano, como agente que cuida, es necesario evaluar de una manera sistemática y global las diferentes dimensiones del cuidador anciano porque, como afirman Morris in Phipps, 
Sands y Marek (10), "[...] en los cuidados gerontológicos y geriátricos, el enfoque de la valoración es el nivel funcional del anciano. [...] cualquier pérdida de capacidades funcionales pueda afectar, de una manera notable, la calidad de vida y el bienestar de los mayores." Como la práctica de la enfermería se debe guiar por principios científicos, surgen las escalas de valoración de la capacidad funcional del anciano [la de Lawton y la de Katz, citadas por Yuaso y Sguizzatto en Netto (11); la de Barthel, referida por Cid-Ruzafa y Damián-Moreno, citados por Imaginário (12), y las escalas de valoración del estado mental y nivel de pensamiento cognitivo [Blessed, citada por Ventura y Bottino in Netto (13)]. Éstas permiten una valoración de las diferentes realidades del anciano, lo que nos permite una valoración multidimensional tanto del anciano cuidador como del anciano que recibe cuidados, teniendo un valor diagnóstico y terapéutico. De acuerdo con Lage (14), ellas "Valoran la función indirectamente a través de las consecuencias derivadas de su pérdida [...] y [...] deben evaluar en función de lo que el sujeto realiza habitualmente y no según las posibilidades teóricas de que pueda realizar determinada tarea”. El diagnóstico de enfermería con la respectiva elaboración del plan de cuidados puede y debe ser auxiliado por el uso de escalas. Sobre las intervenciones de los cuidadores, Brodaty (15) defendió que éstas deben basarse en tres grandes pilares: apoyo psicológico, educación/información y sistemas sociales de apoyo.

El apoyo psicológico y la intervención terapéutica tienen el objeto de promover y mantener la salud mental del cuidador informal anciano, dándole estructuras psicoemocionales para cuidar del otro y de sí. Según Brodaty (15), ese apoyo se consigue a través del intercambio de experiencias y sentimientos (unburdening), del aprendizaje que se realizará con los cuidadores ancianos, del counselling, de la terapia de comprensión, de la terapia cognitiva, de la relajación, de la gestión del estrés y de la promoción del autocuidado. Este apoyo, para Fennel, citado por Brito (16), implicará una definición de las cogniciones del cuidador a través del descubrimiento de nuevas maneras de pensar, nuevas estrategias de coping. Al intervenir se pretende que en el acto de la enseñanza-aprendizaje se consigan desarrollar estrategias que ayuden al anciano a mantener las capacidades cognitivas existentes, nuevas formas de ser y de estar.

Brito (16) dice que las dificultades más frecuentes del cuidador son la falta de información general y el sentimiento de falta de capacidad para realizar diversos procedimientos relacionados con los cuidados diarios del familiar anciano, pudiendo afectar, de manera significativa, su trabajo de cuidar. En este sentido, la educación/información es la segunda intervención terapéutica. Brêtas y Yoshitome, in Duarte y Diogo (17), defienden que será com- petencia de las enfermeras capacitar a los cuidadores para 132 la prestación de cuidados, supervisando periódicamente tales actividades. Esta capacidad del cuidador, a través de la educación, implica, según Brodaty (15), mejorar las aptitudes del cuidador a domicilio en la práctica de los cuidados desarrollando competencias terapéuticas para la resolución de los problemas hasta la planificación, como actuar en situaciones de emergencia, recurrir a apoyos financieros y jurídicos. Según el Consejo Económico y Social (9), los cuidadores necesitan adquirir conocimientos sobre la enfermedad y la dependencia y la enseñanza práctica.

El último aspecto en la intervención terapéutica consiste en informar al cuidador sobre los sistemas sociales de apoyo. El Artículo 84 (Obligación de informar) del Código Deontológico de los Enfermeros (18), en el párrafo d), hace referencia a que los enfermeros asumen el deber de "Informar sobre los recursos a los que la persona puede tener acceso, así como la manera de obtenerlos". Wellman, citado por Paúl (5), define sistema de apoyo como "[...] una red de apoyo social: un conjunto de personas relacionadas con un conjunto de lazos, por ejemplo, relaciones de apoyo emocional, formando una red social, limitada desde el punto de vista analítico, que sólo tiene en cuenta lazos de apoyo y que asume que estos lazos sólo pueden formar una única estructura integrada". Para Berger y Mailloux-Poirier (19), las redes de soporte se clasifican en formales e informales y tienen como fin la ayuda a las personas mayores. "La red de soporte formal [...] está compuesta por organismos de ayuda de servicios gubernamentales, mientras la red informal (o red natural de ayuda) está constituida por el compañero, parientes, amigos y vecinos". Según Paúl (5), esas redes prestan apoyo psicológico, relacionado con el bienestar y apoyo instrumental, es decir, la ayuda física en momentos en que el anciano presenta una deficiencia en sus capacidades funcionales o una pérdida de autonomía, temporal o permanente.

\section{Cuidador informal anciano de Ponta Delgada}

Siguiendo la tendencia nacional, la RAA también ha estado asistiendo a un envejecimiento gradual de la población. Para tener una visión más amplia de este fenómeno hemos elaborado un cuadro ( $\left.\mathrm{n}^{\circ} 1\right)$ que tiene como base los censos de 2001 del SREA (20), donde hemos discriminado el número de ancianos por isla y su respectivo porcentaje teniendo en cuenta la población residente en la isla. Pensamos que no podíamos estudiar al cuidador anciano sin conocer, en líneas generales, su perfil. Por eso, decidimos caracterizar al cuidador anciano en el municipio de Ponta Delgada. El municipio de Ponta Delgada, con un área total de $231,90 \mathrm{~km}^{2}$, está subdividido en $24^{1}$ freguesias. ${ }^{2}$ Este municipio, según los censos de 2001 del 
133 SREA (20), tiene una población residente de 65.854 habitantes, con una densidad de población de 284 habitantes $/ \mathrm{km}^{2}$, con una población de edad igual o superior a 65 años de 7.110 habitantes, que corresponde al 10,8\% de la población.

Decidimos usar tan sólo una manera de recoger la información pretendida, a través del centro de salud de Ponta Delgada (que cuenta con seis unidades de salud y 10 extensiones) por medio de los registros de visitas a domicilio, en los meses de junio y julio de 2006, con el

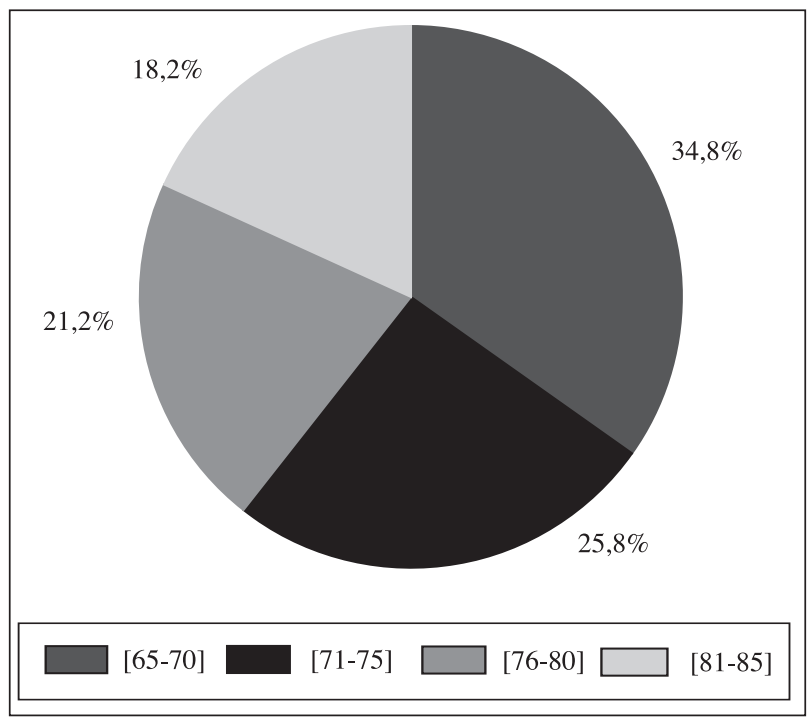

Fig. 1. Edad (años).

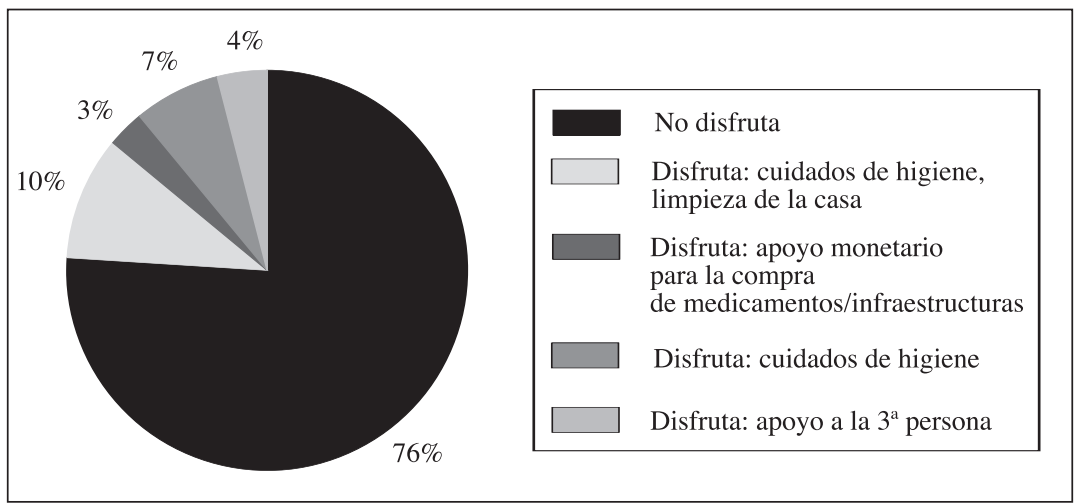

Fig. 2. Apoyo social.

objetivo de identificar a los ancianos objeto de cuidados de enfermería. Con la ayuda de los equipos de enfermería se identificó a los ancianos que tenían como cuidador a otro anciano. Usamos tres criterios de selección para llegar al cuidador anciano: el cuidador tiene una edad igual o superior 65 años; aquel que, sin información específica, presta cuidados al anciano en su ambiente, promoviendo su bienestar y comodidad e intentando mejorar el desempeño de las actividades de la vida cotidiana así como las actividades de la vida social (Fig. 4). cotidiana y, de acuerdo con la clasificación de Agreda (1999) (3), ser una persona mayor sana o una persona mayor de riesgo que cuida a otra persona mayor de riesgo o de un enfermo geriátrico. De los datos recogidos identificamos 66 casos de cuidadores ancianos como está descrito en el Tabla 2.

Para conseguir nuestro objetivo de trazar en líneas generales el perfil del cuidador informal anciano elaboramos un esquema que nos sirvió como orientación durante el análisis de los datos, donde constaban los criterios de caracterización de la muestra (edad, sexo, grado de parentesco, profesión, escolaridad e historial clínico), así como las dimensiones relacionadas con el hecho de ser cuidador (cobertura social, realización de las actividades de la vida cotidiana y relación del anciano cuidado). Nuestro cuidador informal tiene una edad comprendida entre los 65 y los 70 años, y representa el $34,8 \%$ de la muestra, teniendo un grupo de edad de los 81 a los 85 años o más que supone un porcentaje que, creemos, no podemos dejar de destacar por lo que supone para la condición del cuidador anciano, el 18,2\% (Fig. 1). En el género, el sexo femenino es el predominante con el $77,3 \%$ y el grado de parentesco es el de esposa, con el 63,6\%. La profesión que predomina es la de ama de casa con el $62,1 \%$, y se verifica en la muestra, en lo que se refiere escolaridad, que predominan el primer ciclo completo y la ausencia escolaridad con un $40,9 \%$. En las historias clínicas aparece la hipertensión arterial, las enfermedades osteomusculares y la diabetes con porcentajes del 19,9\%, 17,9\% y 10,3\%, respectivamente. En la gran mayoría, el 75,4\%, el cuidador no tiene ayudas sociales (Fig. 2). En la realización de las actividades de la vida cotidiana, el cuidador anciano tiene bajo su responsabilidad los cuidados de alimentación $(26 \%)$, los de higiene y conforto totales $(22 \%)$ y los de eliminación (20\%) (Fig. 3). En la realización de las actividades de la vida social cotidiana se verificó una cierta homogeneidad de los resultados obtenidos sobre la gestión doméstica, en sus tres puntos, con un $14,4 \%$. El acto de cocinar ocupa el segundo lugar con un $12,3 \%$ y la lavandería junto con la administración de medicamentos ocupan el tercer lugar con un 11,8\%

\section{CONCLUSIÓN}

Cuidar a un anciano es siempre una misión que conlleva una serie de vivencias y sentimientos, a veces contradictorios, y de gestión delicada que requiere, por parte del profesional de enfermería, un conocimiento profundo de las diferentes dimensiones que están asociadas al hecho de dar sin recibir, muchas veces.
. 
En este trabajo se ha presentado una reflexión sobre el fenómeno del cuidador informal anciano que cuida a otro anciano, se han intentado analizar las diferentes dimensiones asociadas a él y cuáles son las contribuciones que el enfermero puede desarrollar para que el anciano pueda cuidar manteniendo su espacio para continuar viviendo su envejecimiento de una manera activa y autónoma. Berger y Mailloux-Poirier (19) afirmaron que para ayudar a los ancianos a conservar su autonomía funcional y emotiva hay que favorecer la salud mental y que "Mantener y promover la salud mental exige un tratamiento más preventivo que de curación, y la búsqueda de soluciones de intervención debe tener en cuenta el aspecto emotivo y cognitivo de la persona". En este proceso de búsqueda de un tratamiento preventivo entendemos que tanto el apoyo psicológico como la educación/información son fundamentales en la actividad del enfermero, y que está de acuerdo con lo que el Colegio de los Enfermeros divulga en el Código Deontológico de los En-

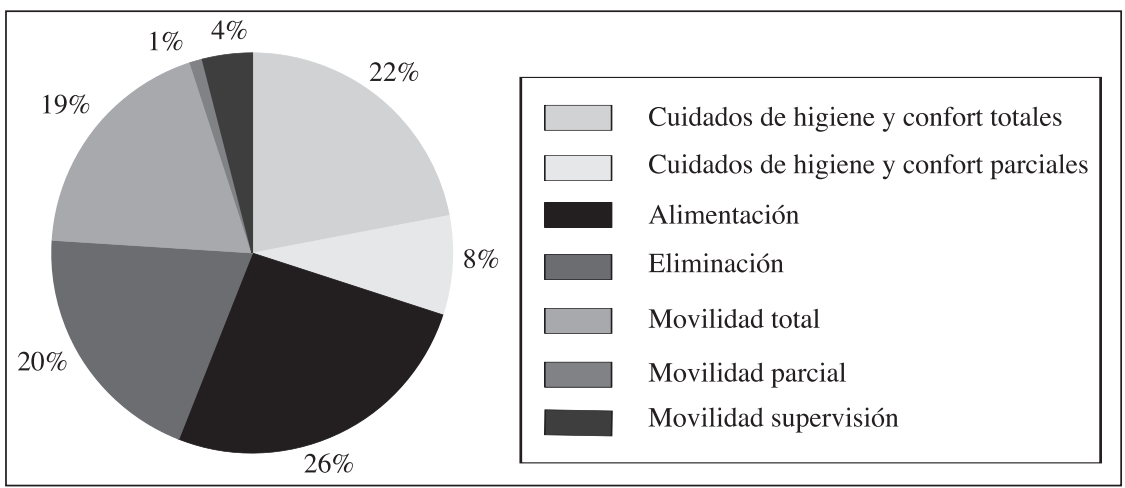

Fig. 3. Actividades de la vida cotidiana.

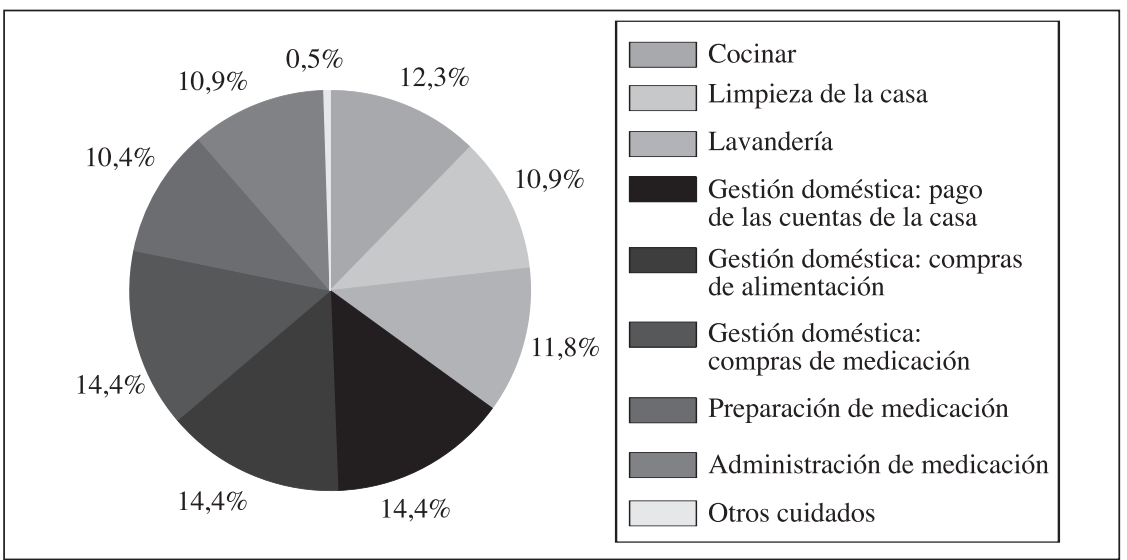

Fig. 4. Actividades de la vida social cotidiana. fermeros (18), en su Artículo 80 (Deber para con la comunidad), que el enfermero es responsable para con la comunidad en la promoción de la salud.

Esto presupone “[...] mejorar la información, educación para la salud y destacar estilos de vida saludables".

\section{BIBLIOGRAFÍA}

1. Costa M. Questôes Demográficas: Repercussōes nos Cuidados de Saúde e na Formação dos Enfermeiros. In O Idoso: Problemas e Realidades. Coimbra: Formasau, 1999; 7-22.

2. Moniz J. A Enfermagem e a Pessoa Idosa: a Prática de Cuidados Como Experiência Formativa. Loures: Lusociência, 2003.

3. Soldevilla Agreda J. Passado, Presente e Futuro da Enfermagem Gerontológica. In O Idoso: Problemas e Realidades. Coimbra: Formasau, 1999; 23-39.

4. Portugal. Serviço Regional de Estatística dos Açores. Estatísticas da Saúde 2004. Angra do Heroísmo: SREA, 2004.

5. Paúl M. Lá Para o Fim da Vida. Coimbra: Livraria Almedina, 1997.

6. Collière M. Promover a Vida. Lisboa: Sindicato dos Enfermeiros Portugueses, 1989.

7. Zimerman Guite. Velhice: Aspectos Biopsicossociais. Porto Alegre: Artmed Editora, 2000.

8. Lurdes S, Storani M. Demência: Impacto para a Família e a Sociedade. In NETTO, M. Gerontologia: A Velhice e o Envelhecimento em Visão Globalizada. São Paulo: Atheneu, 1999; 146-59.

9. Conselho Económico e Social. Responsabilidade Familiar Pelos Dependentes Idosos Nos Países das Comunidades Europeias. Lisboa, 1994.

10. Morris D. A Prática de Enfermagem Junto do Idoso. In Phipps W, Sands J, Marek J. Enfermagem Médico Cirúrgica: Conceitos e Prática Clínica. 6a ed. Vol. I Loures: Lusociência, 2003; 81-105.
11. Yuaso D, Sguizzatto G. Fisioterapia em Pacientes Idosos. In NETTO, M. Gerontologia: A Velhice e o Envelhecimento em Visão Globalizada. São Paulo: Atheneu, 1999; 331-47.

12. Imaginário C. O Idoso Dependente em Contexto Familiar: Uma Análise da Visão da Família e do Cuidador Principal. Coimbra: Formasau, 2004.

13. Ventura M; Bottino C. Avaliação Cognitiva em Pacientes Idosos. In Netto, M. Gerontologia: A Velhice e o Envelhecimento em Visão Globalizada. São Paulo: Atheneu, 1999; 174-89.

14. LAGE I. Aproximação ao seu estado de saúde. Nursing 2005; 195; 612.

15. Brodaty H. Interventions with caregivers. [online]. Disponible en: http://www.malattiemetaboliche.it/articoli/vol4no4a.htm.

16. Brito L. A Saúde Mental dos Prestadores de Cuidados a Familiares Idosos. Coimbra: Quarteto Editora, 2002.

17. Brêtas A, Yoshitome A. Conversando Com Quem Gosta de Cuidar de Idosos no Domicílio. In Duarte Y, Diogo M. Atendimento Domiciliar: Um Enfoque Gerontológico. São Paulo: Editora Atheneu, 2000; 111-3.

18. Ordem dos enfermeiros-Código Deontológico do Enfermeiro: Anotaçôes e Comentários. 2003.

19. Berger L, Mailloux-Poirier D. Pessoas Idosas-uma abordagem global. Lisboa: Lusodidacta, 1995.

20. Portugal. Secretaria Regional de Estatística dos Açores. Recenseamento da População e Habitação. Angra do Heroísmo: SREA, 2003. 